\title{
Incorporation of Potential User Feedback to Inform the Design of a Prototype Integrated Diabetes Management System
}

\author{
Sunetra Bane, MPH \\ Partners Healthcare, Pivot Labs, Boston, MA, United States
}

Corresponding Author:

Sunetra Bane, MPH

Partners Healthcare

Pivot Labs

25 New Chardon Street, Suite 300

Boston, MA, 02114

United States

Phone: 6177242724

Email: sunetrabane@gmail.com

\begin{abstract}
Background: Type 2 diabetes (T2D) is a complex and burdensome chronic disease. Most patients, including uncontrolled (HbA1c > 7\%) patients, only see their Health Care Provider (HCP) once every three months. Thus, a tremendous amount of patient self-management is required outside the clinic to maintain blood glucose (BG) levels within a healthy range and prevent worsening of the disease. Additionally, HCP's overseeing diabetes care often rely on patient self-report and lack key data about how well the patient is managing the condition. We hypothesized that the development of an Integrated Diabetes Management (IDM) system which helps patients better track their BG, insulin and diet would support patient self-management. By sharing a data summary with HCPs through an EMR-integrated, web-based portal, the patient's HCP could would have access to data to help inform treatment decisions and patient education strategies to improve outcomes.
\end{abstract}

Objective: The goal of this design research was to better understand how an IDM platform might support patient self-management and care, and, based on this data, create a prioritized list of requirements for a prototype of the system.

Methods: We used one-hour, semi-structured, in-person interviews and observations with HCPs $(n=18)$ and T2D patients ( $n=14$, recruited via HCP referral). Interview and observation data (via summary notes and transcripts) were analyzed, coding for pain points and workflows. Personas and journey-maps of patients and clinicians were created to inform product design. Pain points and unmet needs were categorized by task and informed the design of features in the IDM system. High fidelity paper prototypes of the solution were tested with HCPs and patients to gather feedback on design options.

Results: HCPs interviewed ranged from MDs $(n=4)$, NPs $(n=7)$, RNs $(n=3)$, CDEs/RDs $(n=2)$ and health coaches $(n=2)$. For HCPs, tasks along the patient journey consist of diagnosis, training, titration, follow-up, and long-term maintenance. Titration and follow-up are the most resource intensive, with the largest number of tasks falling to RNs and NPs. Key HCPs needs included a way to better track patients' behaviors and a way to illustrate the relationship between diet, physical activity and BG/insulin readings to educate patients, improving communication between the patient and their HCP. Patient demographics were predominantly male (57\%), between 50-59 years old (36\%), high-school educated (57\%), and split 50/50 between Android and iOS users. Fifty percent of patients had been diagnosed 1-5 years prior to interview, and 58\% only used long-acting insulin. Patients had the greatest need for help with self-management of their T2D, including reminders and automatic logging of BG and insulin dose, simplified meal and exercise logging, and the ability to share this information with their HCPs.

Conclusions: Design research with T2D patients and HCPs was critical to inform the design of a prototype IDM system. User feedback was incorporated to generate an IDM system which included a patient mobile application, BT-connected insulin event capture device, BT-glucose meter, daily meal log, physical activity tracker, and an HCP clinical portal.

(iproc 2019;5(1):e16200) doi: $10.2196 / 16200$

\section{KEYWORDS}

diabetes mellitus, type 2; human-centered design; user research; product design 
Edited by J Brown; this is a non-peer-reviewed article. Submitted 17.09.19; accepted 17.09.19; published 02.10.19.

Please cite as:

Bane $S$

Incorporation of Potential User Feedback to Inform the Design of a Prototype Integrated Diabetes Management System iproc 2019;5(1):e16200

URL: http://www.iproc.org/2019/1/e16200/

doi: $\underline{10.2196 / 16200}$

PMID:

(O) Sunetra Bane. Originally published in Iproceedings (http://www.iproc.org), 02.10.2019 This is an open-access article distributed under the terms of the Creative Commons Attribution License (https://creativecommons.org/licenses/by/4.0/), which permits unrestricted use, distribution, and reproduction in any medium, provided the original work, first published in Iproceedings, is properly cited. The complete bibliographic information, a link to the original publication on http://www.iproc.org/, as well as this copyright and license information must be included. 